\title{
Economic dynamics with financial fragility and mean-field interaction: a model
}

\author{
C. Di Guilmi a,*, M. Gallegati ${ }^{\text {a, }}$, S. Landini ${ }^{\text {b }}$ \\ ${ }^{a}$ Department of Economics, Università Politecnica delle Marche, Piazzale Martelli \\ 8, 60121 Ancona, Italy \\ ${ }^{\mathrm{b}}$ IRES Piemonte - Istituto Ricerche Economiche e Sociali del Piemonte, Via Nizza \\ 18, 10125 Turin, Italy
}

\begin{abstract}
Following the statistical mechanics methodology, firstly introduced in macroeconomics by Aoki $[1,2,3]$, we provide some insights to the well known works of (author?) [7, 6]. Specifically, we reach analytically a closed form solution of their models overcoming the aggregation problem. The key idea is to represent the economy as an evolving complex system, composed by heterogeneous interacting agents, that can partitioned into a space of macroscopic states. This meso level of aggregation permits to adopt mean field interaction modeling and master equation techniques.
\end{abstract}

Key words: Complex dynamics, master equation, financial fragility, mean field interaction, heterogeneity

PACS: 05.10.Gg, 89.65, 89.75.Fb

\section{Introduction}

Aim of the present work is to replicate the mechanism of the models introduced in [6] and [4] and to resettle them in a dynamic stochastic framework, as defined by Aoki [1, 2, 3]. The quantitative previsions of [4] (and of its developments [12]) replicate a number of stylized facts, strengthening the idea that the economy would be better represented as a complex dynamical system rather than a mere sum of identical and perfect informed agents. Any

\footnotetext{
* Corresponding author: Corrado Di Guilmi, Department of Economics, Università Politecnica delle Marche, Piazzale Martelli 8, 60121 Ancona, Italy. Email address: c.diguilmi@univpm.it.
} 
attempt to resolve analytically this kind of models must face the aggregation issue, since the problem of how to sum up heterogeneous and evolving agents cannot be dealt with the consuete tools of the economist [14]. Masanao Aoki [1, 2, 3] managed to remove the representative agent hypothesis, introducing in economics the ground-breaking concept of mean-field interaction, that makes feasible the analytical aggregation of heterogeneous agents, replacing the unrealistic mechanic determinism of mainstream framework with a set stochastic tools borrowed from statistical mechanics. Mean-field interaction can be defined as the average interaction model that substitutes all the relations among agents that, otherwise, could not be analytically treated[5]. All the agents are clusterized in a pre-defined set of states, basing on one particular feature (the micro-state, e.g. the level of production for each firm) that determines the characteristics and the evolution of the aggregate (the macro-state, e.g. the total level of output). The accent is not on the single agent, but on the number or proportion of agents that occupy a certain state at a certain time. These levels are governed by a stochastic law, that defines also the functional of the probability distributions of aggregate variables and, if existing, their equilibrium distributions. The structure of the work is the following: firstly, we specify the hypothesis for the stochastic structure of the system (section 2 and for the firms that compose it (section 3 )); then (section 44), we develop the probabilistic framework, setting the dynamical instruments needed for the aggregation.

\section{Structure of the system and definition of states}

We set up a model in continuous time for a system of heterogeneous and interacting agents, partitioned into groups or states. In this paragraph we state the hypothesis that are at the root of this stochastic dynamics, and in particular the definition and the structure of the states. Our system is articulated in two states. This partition permits to isolate the effect of bankruptcy costs on the aggregate dynamics. Moreover, the application of recently proposed methods for systems with higher order of states [20] would entail a too high price in terms of computational complication respect to the expected improvement in the realism of the model. Along time, a single firm can be in one of the two states, depending on its financial soundness, proxied by the equity ratio (the ratio among the net worth and the total assets). Therefore there are two types of firms: the "good" firms, that have a high equity ratio, and the "bad" firms, that have a low equity ratio that exposes them to the risk of demise 1 . System works in countinuos time [10]: $t \in \mathbb{T} \subset \mathbb{R}_{+}$.

The economy is populated by a fixed number of firms $N=N(t): \forall t \in \mathbb{T}$, each indexed by $i$ for any given time.

$\overline{1}$ To be complete, there is also a third type, the "ugly" firms, i.e. the failed ones, as explained in the following. 
The system's vector of states $\underline{\omega}$ is identified by the financial condition of the firm:

$$
\underline{\omega}(t)=\left\{\omega_{i}(t)=H V\left(a_{i}(t) \mid \bar{a}\right) \forall i \leq N\right\} \quad: \quad H V\left(a_{i}(t) \mid \bar{a}\right)=\left\{\begin{array}{l}
1 \Longleftrightarrow a_{i}(t)<\bar{a} \\
0 \Longleftrightarrow a_{i}(t) \geq \bar{a}
\end{array}\right.
$$

where $\bar{a}$ represents the threshold of equity ratio, that individuates firms that are in a critical financial situation, and, therefore, for which the probability of bankruptcy is bigger than 0 and where $a_{i}(t)$ is the equity ratio for firm $i$ at time $t$.

In what follows we set $a_{1}(t)=x$ for firms which equity ratio is under the threshold and $a_{2}(t)=y$ for firms which equity ratio is over the threshold.

The cardinality of the $j$-th state, to say the number of firms in state $j=0,1$ is given by

$$
\left.\begin{array}{l}
\operatorname{card} N_{1}(t)=\#\left\{\omega_{i}(t)=1 \forall i \in I\right\}=N_{1}(t) \\
N_{0}(t)=N-N_{1}(t)
\end{array}\right\} \Rightarrow \underline{N}(t)=\left(N_{0}(t), N_{1}(t)\right)(1)
$$

By assumption, the dynamics of the occupation number $N_{j}$ follows a continuous time jump Markov process, defined over a state space $\Omega=(x, y)$ equipped with the following counting measure $N_{(.)}():. \Omega \times \mathbb{T} \rightarrow \mathbb{N}$, so that $\left(\Omega, N_{(.)}().\right)$ is a countable sample space. In the following we indicate with $x$ the case of firms with equity ratio below the threshold $\bar{a}$ and with $y$ the alternative case:

$$
\omega=x \Leftrightarrow \omega_{i}(t)=1 \vee \omega=y \Leftrightarrow \omega_{i}(t)=0
$$

such that $N_{(.)}($.$) evaluates the cardinality of microstates: N_{(\omega)}(t)=N_{1}(t) \Longleftrightarrow$ $\omega=x$ and $N_{(\omega)}=N_{0}(t) \Longleftrightarrow \omega=y$. The relative frequency of firms is indicated in small letters: $n_{k}=N_{k} / N$. A-priori probability of $\omega=1$ is indicated by $\eta$ :

$$
p(\omega=x)=\eta \Leftrightarrow p(\omega=y)=1-\eta
$$

A firm entries into the system in state $x$ and fails (and thus exits from the system) only if it is in state $x$ (i. e. if its probability of bankruptcy is bigger than 0 ); in order to maintain constant the number of firms $N$ we assume that each bankrupted firm is immediately substituted by a new one. Therefore failures of firms do not modify the value of $N_{1}:=N(x)$. Firms move from $x$ 
to $y$ or vice versa according to the following transition rates:

$$
\begin{aligned}
& b\left(N_{j}\right)=r\left(N_{j}+1 \mid N_{j}\right)=\zeta \frac{N-N_{j}}{N}(1-\eta) \\
& d\left(N_{j}\right)=l\left(N_{j}-1 \mid N_{j}\right)=\iota \frac{N_{j}}{N} \eta
\end{aligned}
$$

where $\zeta$ is the transition probability from state $y$ to $x$ (firms whose financial position is deteriorated from a period to another, with equity ratio that becomes lower than $\bar{a}$ ) and $\iota$ the probability of the inverse transition (firms whose equity ratio improved becoming bigger than $\bar{a}$ ). Having already indicated with $N^{j}$ the occupation number of firms in state $j$, transition rates can be evaluated according to:

$$
\begin{aligned}
& b\left(N_{j}\right)=r\left(N_{j}+1 \mid N_{j}\right)=\lambda\left(N-N_{j}\right): \lambda=\zeta(1-\eta) \\
& d\left(N_{j}\right)=l\left(N_{j}-1 \mid N_{j}\right)=\gamma\left(N_{j}\right): \gamma=\iota \eta
\end{aligned}
$$

In statistical mechanics terms, this kind of system can be defined as a statistical ensemble with conservative cardinality, described by a continuous time Markov process over a discrete state space with the structure of a birth-death process.

\section{The Firms}

\subsection{Hypothesis}

The assumptions regarding firms are the same of the original models of (author?) [6, 7], as modified by [4], except for the following specifications. Respect to the theoretical construction proposed by [4] this model does not consider any credit market. There is no direct but a mean-field interaction. Differently from [4] the total number of firms $N$ does not change over time. Firms are identical within each state. The production function of a generic firm $i$ is:

$$
q_{i}(t)=2\left(k_{i}(t)\right)^{1 / 2}
$$

where $k$ is the physical capital and $q$ is the physical output. It follows that the demand of capital function for a single firms will be equal to:

$$
k_{i}(t)=\frac{1}{2}\left(q_{i}(t)\right)^{2}
$$


The multiplicative shock price $\tilde{u_{i}(t)}$ has uniform distribution with support $[0.75 ; 1.25]$ and $\mathbb{E}(\tilde{u})=1$. Given all the above specified hypothesis, the profit function for a generic firm $i$ can be expressed by:

$$
\pi_{i}(t)=P(t) \tilde{u}_{i}(t) q_{i}(t)-r_{i}(t) K_{i}(t)
$$

Once a firm get failed, it faces bankruptcy $\operatorname{costs} C_{i}(t) \mu$ growing with the size of firm, proxied by the volume of its sales, and quantified by:

$$
C_{i}(t)=c\left(P_{i}(t) q(t)_{i}\right)^{2}=c\left(P(t) u_{i}(t) q_{i}(t)\right)^{2} \quad: \quad 0<c<1
$$

\subsubsection{Transition probabilities}

A firm goes bankrupted if it "consumes" all its own capital, then $A_{i} \leq 0$. So that, analogously with [4], we can express the bankruptcy condition as a function of the price shock:

$$
\tilde{u}\left(t^{\prime}\right) \leq\left(\frac{P(t)}{P\left(t^{\prime}\right)}\right)\left(r K_{i}(t) / q_{i}(t)-a_{1}(t) \frac{K_{i}(t)}{P\left(t^{\prime}\right) q_{i}(t)}\right) \equiv \bar{u}_{i}\left(t^{\prime}\right)
$$

Substituting equation 6 into the above expression and, without loss of generality, normalizing reference price $P(t)=P\left(t^{\prime}\right)$ to 1 , it is possible to simplify the r.h.s. of the above equation:

$$
\bar{u}\left(t^{\prime}\right) \equiv \frac{q_{1}(t)}{2}\left(r-a_{1}(t)\right)
$$

Recalling that the random variable $\tilde{u}$ has support $[0.75 ; 1.25]$, the critical thresholds of shock prices for having bankruptcy will be:

$$
\left\{\begin{array}{l}
\bar{u}=0.75 \Longleftrightarrow \tilde{u}_{i}(t)<0.75 \\
\bar{u} \in(0.75 ; 1.25) \Longleftrightarrow 0.75<\tilde{u}_{i}(t)<1.25 \\
\bar{u}=1.25 \Longleftrightarrow \tilde{u}_{i}(t)>1.25
\end{array}\right.
$$

Then, it is possible to indicate the probability $\mu(t)$ of failure for a firm as a function of $\tilde{u}(t)$ :

$$
\mu(t)=F(\tilde{u}(t))=p(\tilde{u}(t) \leq \bar{u}(t))=\frac{\bar{u}(t)-0.75}{0.5}=2 \bar{u}(t)-1.5
$$

Equation (12) permits to determine endogenously the threshold $\bar{a}$ : indeed it can be interpreted as the minimum value of equity ratio which ensures the 
surviving of the firm, i. e. for which the probability of bankruptcy is equal to zero, and, therefore, it can be expressed as:

$$
\bar{a}\left(t^{\prime}\right)=r-\frac{1.5}{q_{1}(t)}
$$

With an analogue procedure, we can specify the transition probabilities $\zeta$ and $\iota$ as dependent variables of the price shock $u_{i}(t)$. Indicating the critical values, respectively with $\bar{u}_{i, \zeta}(t)$ and $\bar{u}_{i, \iota}(t)$ we obtain:

$$
\begin{aligned}
& \tilde{u}_{i}(t) \leq \frac{q_{0}(t)}{2}\left(r+\bar{a}(t)-a_{0}(t)\right) \equiv \bar{u}_{\zeta}(t) \\
& \tilde{u}_{i}(t)>\frac{q_{1}(t)}{2}\left(r+\bar{a}(t)-a_{1}(t)\right) \equiv \bar{u}_{\iota}(t)
\end{aligned}
$$

since the thresholds, that for the 11 is equal to 0 , here become equal to, respectively $\left(\bar{a}(t)-a_{0}(t)\right)$ and $\left(a_{1}(t)-\bar{a}(t)\right)$. The range of variation of the two thresholds is truncated as done in 10. It is straightforward now to get the transition probability for each state:

$$
\begin{array}{r}
\zeta(t)=p\left(\tilde{u}(t) \leq \bar{u}_{\zeta}(t)\right)=2 \bar{u}_{\zeta}(t)-1.5 \\
\iota(t)=1-p\left(\tilde{u}(t) \leq \bar{u}_{\iota}(t)\right)=-2 \bar{u}_{\iota}(t)+2.5
\end{array}
$$

\subsection{Firms object function.}

A firm decide the optimal quantity to produce in order to maximize its profit, using the information at its disposal. Under the stated hypothesis the object function of a generic firm $i$ can be then expressed as:

$$
\max _{q_{i}(t)} F\left(q_{i}(t)\right):=\left\{\mathbb{E}\left[P(t) u_{i}\left(t^{\prime}\right) q_{i}(t)\right]-r K_{i}(t)-C_{i}(t) \mu\left(t^{\prime}\right)\right\}
$$

We suppose that firms take into consideration the present level of failure probability, therefore that $\mu\left(t^{\prime}\right)=\mu(t)$, and that $P^{e}\left(t^{\prime}\right)=P\left(t^{\prime}\right)=P(t)=1$ without loss of generality. The first order condition is:

$$
1-r q_{i}(t)-2 c q_{i}(t) \mu(t)=0
$$

Consequently we get two different optimal levels of production for firms in state $x$ and for firms in state $y$, respectively:

$$
\begin{array}{r}
q_{1}^{*}=(r+2 c \mu)^{-1} \\
q_{2}^{*}=r^{-1}
\end{array}
$$


since $\mu=0$ for firms in state $y$.

\section{Stochastic inference}

\subsection{Master equation and stationary points}

In order to specify the dynamics of the joint probabilities, and, by this way, the stochastid 2 evolution of the system, following [2, chap. 3], [8, page 252] and [11, page 62] we make use of the following master equation:

$$
\begin{aligned}
\frac{d P\left(N_{k}, t\right)}{d t} & =b\left(N_{k-1}\right) P\left(N_{k-1}\right)+d\left(N_{k+1}\right) P\left(N_{k+1}\right)+ \\
& -\left\{\left[\left(b\left(N_{k}\right)+d\left(N_{k}\right)\right) P\left(N_{k}\right)\right]\right\}
\end{aligned}
$$

with boundary conditions:

$$
\left\{\begin{array}{l}
P(N, t)=b\left(N^{1}\right) P\left(N^{1}-1, t\right)+d(N) P(N, t) \\
P(0, t)=b(1) P(1, t)+d(0) P(0, t)
\end{array}\right.
$$

These conditions ensure that the distributions functions consider only consistent values, that is to say $N_{1} \in[0 ; N]$ and, therefore, $n_{1} \in[0 ; 1]$. Since an analytical solution for master equations can be obtained only under very specific conditions [23], we solve it with an approximation method, based on led and lag operator 3 . We assume that the number of firms in state $x$ at a given moment is determined by its expected mean $(m)$, the drift, and by an additive fluctuations component of order $N^{-1 / 2}$ around this value, that is to say the spread:

$$
N_{1}=N m+\sqrt{N} s
$$

Solving the modified master equation (see Appendix a) we find an explicit formulation for the macroscopic equation:

$$
\frac{d N_{1}}{d t}=-(\lambda+\gamma) N_{1}+\lambda N
$$

$\overline{2}$ Indeed, "the master equation describes the time evolution of the probability distribution of states, not the time evolution of the states themselves" [2, page 7].

3 For a detailed exposition see [2] or [8]. 
This equation describes the dynamics of the drift. It can be interpretated as the long-run trend of the occupation number and, then, keeping all the other relevant variables unchanged, of the production. Now we can determine the stationary value of $\mathbb{E}\left(n_{k}\right)=m$ and the consequent steady state equilibrium of the economy, simply setting the r.h.s. of the (20) to 0:

$$
m^{*}=\frac{\lambda}{\lambda+\gamma}=n_{1}^{*}
$$

Once demonstrated the existence of a dynamical equilibrium point, a deeper insight on system's dynamic is needed in order to verify the convergence of the dynamics toward the equilibrium distribution. The solution of the differential equation (20) is:

$$
m(t)=m_{0} e^{-(\lambda+\gamma) t}+\frac{\lambda}{\lambda+\gamma}
$$

that, setting an initial point $m_{0}$, yields to:

$$
m(t)=m^{*}+\left(m_{0}-m^{*}\right) e^{-(\lambda+\gamma) t}
$$

that verifies the convergence and the stability of the equilibrium since the second term goes to 0 as $t \rightarrow \infty$.

\subsection{Aggregate output}

The aggregate output of the system will be:

$$
Y(t)=\frac{N_{1}}{r+2 c \mu(t)}+\frac{N_{0}}{r}
$$

Keeping constant all the other variables, dynamic fluctuations in the level of $Y(t)$ are due to the changing in the levels of $N_{1}$ and $N_{0}$. Once quantified the equilibrium distribution of the drift, we are able now to obtain the steady state value of aggregate production:

$$
Y^{e}=N\left[\frac{1}{r}-\frac{\lambda}{\lambda+\gamma} \frac{2 c \mu}{r(r+2 c \mu)}\right]=N\left[\frac{1}{r}-\frac{\lambda}{\lambda+\gamma}\left(q_{1}-q_{0}\right)\right]
$$

As can be easily seen the factor inside the brackets of the (25) is equal to

$$
\left[y_{0}-m^{*}\left(y_{1}-y_{0}\right)\right]
$$


that testifies the consistency of the model. Since the aggregate production function depends on $m$, also its dynamic will be convergent to a stationary level. Dynamics comes out to be dependent on the transition rates $\lambda$ and $\gamma$ and on the differences in firms level of production. The dynamics of these factors are studied in the next section.

\subsection{Equilibrium distribution and critical points}

Equating the master equation to 0, it is possible to obtain the Kolmogorov condition that equates the probability fluxes entering in a state with the fluxes coming out from that state. Formally, using equations 4 and 20:

$$
\dot{m}=\zeta \eta(m)-[\iota(1-\eta(m))+\zeta \eta(m)] m
$$

Setting $\dot{m}$ to 0 and rearranging, the stationary configuration of the system can be expressed as:

$$
\dot{m}=0 \Rightarrow \frac{\eta\left(m^{*}\right)}{1-\eta\left(m^{*}\right)}=\frac{\iota m^{*}}{\zeta\left(1-m^{*}\right)}
$$

Being in Markovian space, we can make use of Brook's lemma [15] that defines local characteristic for this kind of chains:

$$
P^{e}\left(N_{k}\right)=P^{e}\left(N_{0}\right)\left(\frac{\iota}{\zeta}\right)\left(\begin{array}{c}
N \\
N_{k}
\end{array}\right) \prod_{j=1}^{k} \frac{\eta\left(N-N_{j}\right)}{\left(1-\eta\left(N_{j}\right)\right)}
$$

By means of Hammersley and Clifford theorem (see [16] as demonstrated in [17]), the stationary probability of the markovian process for $N_{j}$, when detailed balance holds, can be expressed by:

$$
P^{e}\left(N_{j}\right) \propto Z^{-1} e^{-\beta N U\left(N_{j}\right)}
$$

where $U\left(N^{j}\right)$ is the Gibbs potential [9]. The parameter $\beta$ may be interpreted as an inverse measure of the uncertainty of the system. The above formulation allows us to express explicitly the values of the a-priori probabilities:

$$
\begin{array}{r}
\eta\left(N_{j}\right)=N^{-1} e^{\beta g\left(N_{j}\right)} \\
1-\eta\left(N_{j}\right)=N^{-1} e^{-\beta g\left(N_{j}\right)}
\end{array}
$$

so that:

$$
e^{\beta g\left(N_{j}\right)}+e^{-\beta g\left(N_{j}\right)}=N
$$


It is easy to verify that large values of $\beta$ associated with positive values of $g\left(N_{j}\right)$ cause $\eta\left(N_{j}\right)$ to be larger than $1-\eta\left(N_{j}\right)$, making transition from state $y$ to state $x$ more likely to occur than the opposite one. On the other hand, values of $\beta$ close to 0 , make $\eta\left(N_{j}\right)$ close to 0.5 . To get a deeper insight on the meanings of $\beta$ let us carry out the potential equation. In binary models and for great $N$, the equation of the potential is:

$$
U\left(N^{j}\right)=-2 \int_{0}^{N^{j}} g\left(N_{j}\right) d y-\frac{1}{\beta} H(\underline{N})
$$

where $H(\underline{N})$ is the Shannon entropy for the vector of occupation numbers. $g\left(N_{j}\right)$ is a function that evaluates the relative difference in the outcome as a function of $N_{j}$. In intuitive terms, to individuate the stationary points of probability dynamics we need to individuate its peak. $\beta$ is an inverse multiplicative factor for entropy: this implies then that, for very large values of $\beta$, the entropy component does not play any role. On the contrary, as $\beta$ approaches 0 , the weight of the entropy component grows. In terms of the present model, a relative high value of $\beta$ means that the uncertainty in the system is low, with few firms exposed at bankruptcy risk and, due to this, the bankruptcy probability $\mu$ plays a negligible role [1, pp. 55 and following]. Therefore for values of $\beta$ around 0 , and a more relevant volatility in the system, in order to individuate the peak of probability dynamic we need to find the local minimum of the potential. [2] shows that the points in which the potential is minimized are also the critical point of the aggregate dynamics of $p_{j}$. Deriving the potential respect to $N^{j}$ :

$$
g\left(N^{j}\right)=-\frac{1}{2 \beta} \frac{d H\left(N_{j}\right)}{d N_{j}}=-\frac{1}{2 \beta} \ln \left(\frac{N_{j}}{N-N_{j}}\right)
$$

Solving the following MaxEnt problem [21]:

$$
\left\{\begin{array}{l}
\max H\left(N_{1}, N_{0}\right)=-N_{1}(t) \ln \left(N_{1}(t)\right)-N_{0}(t) \ln \left(N_{0}(t)\right) \text { s.t. } \\
N_{1}(t)+N_{0}(t)=N \\
N_{1}(t) y_{1}(t)+N_{0}(t) y_{0}(t)=Y(t)
\end{array}\right.
$$

with suitable Lagrange multipliers equal to, respectively, $\lambda_{1}=1-\alpha$ and $\lambda_{2}=-\beta$ we get a closed solution for $\beta$ :

$$
\beta(t)=\ln \left(-\frac{y_{1}(t)-y \overline{(t)}}{y_{0}(t)-y \overline{(t)}}\right)\left(y_{1}(t)-y_{0}(t)\right)^{-1}
$$


Setting $U^{\prime}=0$ and using equation (35), we get an explicit formulation for $g\left(N_{1}\right)$ in stationary condition:

$$
g\left(N_{1}\right)=\frac{y_{0}-y_{1}}{2}
$$

that quantifies the mean difference (for states) of the outcome.

From equation 33 it follows that the point of local minimal of the potential is given by:

$$
U^{\prime}=0 \Rightarrow e^{2 \beta g\left(N_{j}\right)}=\frac{N_{j}}{N-N_{j}}
$$

that, if the rates of entries and exits are equated, (i. e. if $\iota=\zeta$ ), reproduces exactly the 27. Therefore, making use of the 30 we can write:

$$
N_{j}=\frac{e^{2 \beta g\left(N_{j}\right)}}{e^{2 \beta g\left(N_{j}\right)}+e^{-2 \beta g\left(N_{j}\right)}}
$$

that is the maximum likelihood estimation of the Gibbs distribution of the firms in state $N_{j}$. Let us analyze the different behavior of the stationary distribution for different values of $\beta$.

$\beta \rightarrow \infty$ Given equation [33, the critical points in which the potential is minimized are also the zeros of the function $g\left(N_{j}\right)$ :

$$
U^{\prime}\left(m^{*}\right)=-2 g\left(m^{*}\right)=0
$$

This suggests that $\beta$ may be interpreted as an inverse index of uncertainty [1] . Considering equation 36 .

$$
g\left(N_{j}\right)=0 \Leftrightarrow y_{0}-y_{1}=0
$$

Under the specified conditions, there is no uncertainty in the system, since no firm can go bankrupted and, therefore, the level of production is unambiguously determined. Indeed the value of $\beta$ can go to infinity if $N_{0} \rightarrow N$ or if $\mu \rightarrow 0$, since both situation imply a convergence among the different targets of production at micro level and, then, a minimum degree of uncertainty in the system.

$\beta \rightarrow 0$ In this second case, in order to individuate the critical points of the dynamics, a further deepening is needed. $\beta$ can go to 0 if and only if $\frac{N_{j}}{N-N_{j}} \rightarrow 1$, that is to say, if the system is populated, quite in the same proportion, of firms in state $x$ and of firms in state $y$. But this is not informative 
about the behavior of the $g(N)$ since the first factor of the equation 33 goes to infinity (given that $\beta \rightarrow 0$ ) while the second goes to 0 . The Cox and Miller hazard function [18], with a density function analogous to the one of equal to a standard Brownian motion's first-passage [19], in terms of $m$ can be expressed as:

$$
F(m)=\left[1+e^{-2 \beta m}\right]^{-1} \Rightarrow h(m)=\frac{2 \beta}{1+e^{-2 \beta m}}
$$

Now, we have to calculate the probability that a firm passes from a state to another in response to a small variation in the difference of relative production, conditional on the current difference among $y_{0}$ and $y_{1}$ (quantified by $g(m)$, from equation 36). We can then rewrite the conditional hazard function in the following way:

$$
P\left(v \leq y_{1}-y_{0} \mid m^{*}\right)=\left[1+e^{-2 \beta g\left(m^{*}\right)}\right]^{-1}
$$

and then:

$$
h\left(y_{1}-y_{0} \mid m^{*}\right)=\frac{2 \beta \eta\left(m^{*}\right)}{1+e^{-2 \beta g\left(m^{*}\right)}}
$$

Supposing that $\eta\left(m^{*}\right)=m^{*}$, we finally obtain:

$$
h\left(y_{1}-y_{0} \mid m^{*}\right)=2 \beta m^{*}
$$

Therefore, we may conclude that for values of $\beta$ close to 0 , the critical point of probability dynamics, here found by minimizing the potential, is a value of $m^{*}$ approximately equal to $\beta$ itself. In other words, $\beta$ may be considered the as the conditional hazard rate in the range where $\beta$ is small. The potential then is minimized for a fraction $m^{*}$ of firms in state $x$ when the value of the conditional hazard function is approximately equal to $\beta$.

\section{Concluding remarks}

In this work we apply Aoki's methodology to the model presented in [6] and modified, introducing interacting heterogeneous agents, in [4]. The adoption of the statistical mechanical approach, based on mean field interaction and aggregate stochastic dynamic analysis, leads to a stable analytical solution, without recurring to computer simulations. We start from very general conditions and hypothesis, embodied in a stochastic framework, and, finally, we

obtain an explicit macroscopic equation that describes the evolution of the 
system and its long term equilibrium solution. The dynamics is analyzed by means of master equation solution techniques enriched by the use of MaxEnt and hazard function analysis.

\section{Appendix A}

The master equation has now to be expressed as $\dot{Q}(s)$, a function of $s$, and then becomes:

$$
\dot{P}\left(N_{k}\right)=\frac{\partial Q}{\partial t}-\frac{d s}{d t} \frac{\partial Q}{\partial s}=\dot{Q}(s)
$$

with transition rates reformulated in the following way:

$$
\begin{array}{r}
b(s)=\lambda[N-N m-\sqrt{N} s] \\
d(s)=\gamma[N m+\sqrt{N} s]
\end{array}
$$

Since

$$
\frac{d s}{d t}=-N^{1 / 2} \frac{d m}{d t}
$$

equation (.1) can be expressed as:

$$
\dot{Q}(s)=\frac{\partial Q}{\partial t}-N^{1 / 2} \frac{\partial Q}{\partial s} \dot{m}
$$

We rewrite again now the master equation (17) and the transition rates by means of lead and lag operators. The use of these operators allows to express the master equation in a more treatable form, making the two probability flows (in and out) homogeneous. Specifically the transition probabilities (4) become:

$$
\begin{aligned}
L\left[d\left(N_{k}\right) P\left(N_{k}, t\right)\right] & =d\left(N_{k+1}\right) P\left(N_{k+1}\right) \\
L^{-1}\left[b\left(N_{k}\right) P\left(N_{k}, t\right)\right] & =d\left(N_{k-1}\right) P\left(N_{k-1}\right)
\end{aligned}
$$

so that the master equation will be expressed in this way:

$$
\dot{Q}(s)=(L-1)[d(s) Q(s)]+\left(L^{-1}-1\right)[d(s) Q(s)]
$$

Using the modified transition rates (.6) and expanding the so obtained master 
equation in inverse powers of $s$ to the second order we get:

$$
\begin{aligned}
& N^{-1} \frac{\partial Q}{\partial \tau}-N^{-1 / 2} \frac{d m}{d \tau} \frac{\partial Q}{\partial s}= \\
& N^{-1 / 2}\left(\frac{\partial}{\partial s}\right)[d(s) Q(s)]+N^{-1} \frac{1}{2}\left(\frac{\partial}{\partial s}\right)^{2}[d(s) Q(s)]+ \\
& -N^{-1 / 2}\left(\frac{\partial}{\partial s}\right)[b(s) Q(s)]+N^{-1} \frac{1}{2}\left(\frac{\partial}{\partial s}\right)^{2}[b(s) Q(s)]+\ldots \\
& =N^{-1 / 2}\left(\frac{\partial}{\partial s}\right)[(d(s)-b(s)) Q(s)]+N^{-1} \frac{1}{2}\left(\frac{\partial}{\partial s}\right)[(b(s)+d(s)) Q(s)]+\ldots
\end{aligned}
$$

At this point, in order to match the component of the same orders of powers

of $N$ between and equations (.1) and (.9), we need to rescale the variable $\tau=t N$. Knowing that:

$$
\begin{aligned}
& d(s)-b(s)=(\lambda+\gamma)(N m+\sqrt{N} s)-\lambda N=(\lambda+\gamma) N_{k}-\lambda N \\
& d(s)+b(s)=(\lambda-\gamma)(N m+\sqrt{N} s)+\lambda N=(\lambda-\gamma) N_{k}+\lambda N
\end{aligned}
$$

and taking the derivatives, up to the second order, it is possible to obtain what Aoki defines as diffusion approximation [2]:

$$
\begin{aligned}
& N^{-1} \frac{\partial Q}{\partial \tau}-N^{-1 / 2} \frac{d m}{d \tau} \frac{\partial Q}{\partial s}= \\
& (\lambda+\gamma) Q(s)+N^{-1 / 2}(d(s)-b(s))\left(\frac{\partial}{\partial s}\right) Q(s)+N^{-1} \frac{1}{2}(b(s)+d(s))\left(\frac{\partial}{\partial s}\right) Q(s)
\end{aligned}
$$

Equating the terms of order $N^{-1 / 2}$ for the polynomial identity principle we get:

$$
N^{-1 / 2} \frac{d m}{d t} \frac{\partial Q}{\partial s}=-N^{-1 / 2}(b(s)-d(s))\left(\frac{\partial}{\partial s}\right) Q(s)
$$

\section{References}

[1] Aoki, Masanao, New approaches to macroeconomic modeling, Cambridge University Press, 1996.

[2] Aoki, Masanao, Modeling aggregate behaviour and fluctuations in economics, Cambridge University Press, 2002.

[3] Aoki, Masanao and Yoshikawa, Hiroshi, Reconstructing Macroeconomics, Cambridge University Press, 2006.

[4] Delli Gatti, Domenico and Di Guilmi, Corrado and Gaffeo, Edoardo and Giulioni, Gianfranco and Gallegati, Mauro and Palestrini, Antonio, A new approach to business fluctuations: heterogeneous interacting agents, scaling laws and financial fragility, Journal of Economic Behavior and Organization, 2005, volume 56. 
[5] Opper, M. and Saad, D., Advanced Mean Field Methods: Theory and Practice, The MIT Press. Cambridge, MA, 2001.

[6] Greenwald, Bruce and Stiglitz, Joseph, Financial markets imperfections and business cycles., Quarterly journal of Economics, 1993.

[7] Greenwald, B. and Stiglitz, J., Macroeconomic models with equity and credit rationing., in Information, Capital Markets and Investment, Chicago University Press, Chicago, 1990, ed Hubbard, R.

[8] Landini, Simone, Modellizzazione stocastica di grandezze economiche con un approccio econofisico, University Bicocca, Milan, 2005.

[9] Woess, W., Catene di Markov e teoria del potenziale discreto, Quaderni UMI n.41. Pitagora Editrice Bologna, 1996.

[10] Hinich, Melvin J. and Foster, John and Wild, Phillip, Structural change in macroeconomic time series: A complex systems perspective, Journal of Macroeconomics, 2006.

[11] Kubo, R. and Toda, M. and Hashitume, Statistical Physics II. Non Equilibrium Statistical Mechanics, Springer Verlag Berlin, 1978.

[12] Gallegati, M., and Delli Gatti, D., and Gaffeo, E., and Giulioni, G., and Palestrini, A., Emergent Macroeconomics, Springer, 2007.

[13] Minsky, Hyman, Inflation, recession and economic policy, New York: ME Sharpe, 1982.

[14] Gallegati, M, and Palestrini, A, and Delli Gatti, D, and Scalas, E., Aggregation of heterogeneous interacting agent: the variant representative agent framework, Journal of Economic Interaction and Coordination, 2006.

[15] Brook, D., On the distinction between the conditional probability and the joint probability approaches in the specification of nearest-neighbour systems., Biometrica, 1964, vol 51, pages 481-483.

[16] Hammersley, J. M. and Clifford, P., Markov field on finite graphs and lattices, unpublished, 1971.

[17] P. Clifford, Markov random fields in statistics, in "Disorder in Physical Systems. A Volume in Honour of John M. Hammersley", "Clarendon Press", 1990.

[18] Cox, D. and Miller, H., The Theory of Stochastic Processes, Chapman and Hall, 1996.

[19] Grimmett, G.R. and Stirzaker, D.R., Probability and Random Processes, Clarendon Press, Oxford, 1992.

[20] de La Lama, M. S. and Szendro, I. G. and Iglesias, J. R. and Wio, H. S., Van Kampen's expansion approach in an opinion formation model, The European Physical Journal B, 2006.

[21] Jaynes, E. T., Information Theory and Statistical Mechanics, Phys. Rev., 1957, vol. 106.

[22] Landini, S. and Uberti, M., A statistical mechanic view of macrodynamics in economics, forthcoming, 2007.

[23] Risken, H, Fokker-Planck equation. Method of solutions and applications., Berlin: Springer Verlag, 1989. 